\title{
日 lugar de las emociones en la constitución social de lo psíquico: $\boxminus$ aporte de Vigotski
}

\author{
Fernando L. González Rey *
}

RESUMO: O artigo analisa o lugar das emoções na constituição social do psíquico, destacando, sobretudo, a contribuição de Vigotski. O texto enfatiza, particularmente, duas das idéias que mais impacto exerceram na reconstrução da psicologia por Vigotski: o desenvolvimento das funções psíquicas na atividade e a mediação dos signos na constituição dessas funções. O conceito de "situação social do desenvolvimento" revela a preocupação de Vigotski em integrar a riqueza dos processos internos, constituídos na história anterior do sujeito, com as influências que caracterizam cada um dos momentos sociais do desenvolvimento. Destaca-se também a importância que a "vivência" e a categoria de "necessidade" têm no pensamento de Vigotski. Finalmente, é analisada a questão da "personalidade" como construção teórica que reconhece ontologicamente a subjetividade individual, entendida como um processo de um sujeito que existe socialmente.

Palavras-chave: Emoção em Vigotski, situação social do desenvolvimento, vivência, necessidade, personalidade

\section{Introducción}

Después del origen experimental de la psicología en Leipzig en 1879, y a pesar de que la gran mayoria de los estudiosos relevantes de la psicología norteameriana de principios de siglo se formó en el

\footnotetext{
* Pesquisador associado da UnB.
} 
laboratorio de Wundt (Tichener, Cattell, Stanley Hall y el propio Mead), el desarrollo de la psicología norteamericana en los comienzos del siglo XX tomó el camino de la identificación absoluta con el modelo de las ciencias naturales, y esa marca fue extraordinariamente fuerte en el desarrollo de la psicología mundial.

La comprensión de la psique fue adquiriendo cada vez más un color naturalista, el que de una forma $u$ otra se expresaba en corrientes tan diferentes como el psicoanálisis y el conductismo en lo que respecta a la construcción teórica de su objeto. El conductismo enfatiza un objeto común en el estudio del hombre y de los animales: el comportamiento, desarrollando leyes análogas para su estudio en el mundo animal y humano, lo que se acompañó de laboratorios llenos de palomas en las facultades de psicología de la mayor parte del mundo; por su parte, Freud enfatiza el estudio del inconsciente, entre otras cosas, porque es la parte de la mente directamente relacionada con las pulsiones, lo que le permite evitar como aspecto central de la teoría la cuestión de la conciencia, la cual tenía una fuerte connotación mentalista en la época.

Esta visión biologizadora cede su lugar a una representación social de lo psíquico, la cual se legitima con gran fuerza con la aparición de la psicología soviética, fuertemente influida por el marxismo. Fueron muy importantes en aquella época los trabajos de dos de sus pioneros, L.S. Vigotski y S.L. Rubinstein, sin embargo, por razones que escapan a los objetivos del presente trabajo, la influencia de Vigotski se extendió con mayor fuerza, llegando a convertirse en un referente importante de diferentes corrientes del pensamiento en la psicología occidental, entre las que se destaca el enfoque sociocultural. El trabajo de Vigotski, como se expresa a lo largo de toda su obra, estuvo muy influido por diferentes autores de la psicología de su tiempo, entre los cuales se encuentran muchos que habían enfatizado el lugar de lo social, a pesar de que lo hubieran hecho desde posiciones diferentes, desde Adler hasta Piaget, Lewin y Janet.

Ya autores pragmáticos como C. Peirce y G.H. Mead, quienes habían enfatizado el papel del lenguaje, se habían planteado el carácter social de la psique; sin embargo, ambos no constituyeron un referente para la psicología norteamericana de comienzos del siglo XX, totalmente dominada por una visión naturalista y positivista, que terminó anulando la importante influencia de autores como James y Dewey, quienes, a pesar de haber representado un importante antecedente del conductismo, nunca habrían concordado con el camino posterior que sus influencias tuvieron. 
El marxismo en la Unión Soviética no tuvo una influencia inmediata en el desarrollo de una concepción cultural de la psique, la cual fue un logro de los autores soviéticos mencionados, que se fue desarrollando después de múltiples batallas teóricas en relación a la comprensión biologicista de lo psíquico, concretada en la reflexología pavloviana. Sin embargo, la comprensión del carácter cultural de lo psíquico no representó sólo la adscripción a una nueva visión del hombre para los psicólogos soviéticos, sino que implicó una reconstrucción profunda de las bases sobre las cuales se desarrollaba la psicología de la época, a pesar de que Vigotski, particularmente, mantuvo un fructífero diálogo con aquella, de forma especial con autores como Piaget, Lewin y Stern.

En este proceso de reconstrucción de la psicología aparecieron dos ideas muy fuertes, que tuvieron profundo impacto en el desarrollo de la psicología: el desarrollo de las funciones psíquicas en la actividad, estudiadas tanto por Vigotski como por Rubinstein, y la idea de la mediatización de las funciones psicológicas superiores por los signos. Estas ideas fueron esenciales en la comprensión del carácter cultural e histórico del psiquismo humano, y abrieron el camino para una psicología soviética donde encontraron una rápida extensión y desarrollo a través de diferentes grupos de trabajo.

En los trabajos, tanto de Vigotski, como de Rubinstein, se presentó una serie de ideas muy comprometidas con el desarrollo de una ontología diferente de los procesos psíquicos, las cuales se acercaban mucho a la forma en que hoy se presenta el concepto de subjetividad desde diferentes perspectivas. Entre estas ideas estaban la integración de lo cognitivo y lo afectivo en formas complejas de organización de la personalidad humana, la superación de la dicotomía entre lo externo y lo interno y entre lo social y lo individual, así como el planteamiento de formas de organización complejas y procesuales de la psique, como fueron el concepto de personalidad y de funciones psíquicas superiores. También las categorías de significacíon y sentido de Vigotski resultaban promisorias para el desarrollo del tema de la subjetividad.

Sin embargo, como mostró la historia posterior, las ideas de estos autores, esencialmente de Vigotski, se vieron desarrolladas por una psicología que, enfatizando lo social, terminó objetivando la comprensión de lo psíquico, como lo fue la Teoría de la Actividad de A.N. Leontiev, sobre la cual hemos desarrollado diferente análisis críticos en obras anteriores. ${ }^{1}$ Esta teoría se convirtió en una especie de psicología oficial que pasó a ser un referente para evaluar el carácter "marxista" de la producción psicológica, lo que determinó la reificación del término, y su consecuente pérdida de va- 
lor heurístico. En este contexto, ocurrió una vuelta al positivismo metodológico, reforzándose el uso del experimento en la investigación psicológica, que se orientó preferentemente al estudio de procesos sensoriales y cognitivos en los marcos de la Teoría de la Actividad.

Esta Teoría representó un nuevo momento de una vieja tendencia, que la Psicología Soviética, por el contexto ideológico en que se desarrolló, no pudo superar nunca: su énfasis en identificar la materialidad de lo psíquico en formas objetivas, biológicas o sociales. Esto nunca le permitió a los psicólogos soviéticos la construcción teórica de una visión ontológica diferente de la psique partiendo de la definición de su carácter cultural.

Un tema muy mal tratado desde la perspectiva psicológica fue el de la emoción. La psicología naturalista fue incapaz de desarrollar una visión diferente de la emoción, específica de la condición cultural del hombre, de ahí la sustancialización de las fuentes de las emociones en tendencias universales como la pulsión, o el desarrollo de taxonomías rígidas e invariables de necesidades, que actuaban como los espacios en los que se podían ubicar los diferentes tipos de emociones. La fuerza de la psicología cognitiva en el desarrollo de la psicología en los años 50, el impacto de la lingüística que aparece con particular fuerza después de la Segunda Guerra Mundial y la influencia de la semiótica, llevaron a identificar lo social con las dimesiones del lenguaje y las significaciones, planteándose la emoción sólo en su función de significación, lo que, desde mi punto de vista, ha significado un nuevo momento en la representación unilateral de la subjetividad humana.

Ante el hecho de que las emociones no han dejado de ser nunca un epifenómeno de otros procesos, y han aparecido siempre asociadas a lo biológico o a lo social, pero sin un status propio, hemos decidido recuperar la memoria sobre su uso en un conjunto de autores, dedicando este artículo a la recuperación de esta memoria en los trabajos de Vigotski.

\section{Las emociones en los trabajos de Lev S. Vigotski}

El uso del término emoción aparece realmente disperso en los trabajos de Vigotski, pues él no se dedicó de forma específica al estudio de este tema; sin embargo, fue un término que acompañó explicitamente su representación sobre la psicología, y del que dejó constancia en muchos de sus trabajos.

Antes de asumir la conciencia como su objeto de estudio, Vigotski se había planteado el tema del desarrollo como central en su obra, y en 
relación al mismo introdujo un concepto poco citado entre los estudiosos actuales de su obra, que fue el de "situación social del desarrollo". Vigotski entendía la situación social del desarrollo como

\begin{abstract}
(...) aquella combinación especial de los procesos internos del desarrollo y de las condiciones externas, que es típica en cada etapa y que condiciona también la dinámica del desarrollo psíquico durante el correspondiente periodo evolutivo y las nuevas formaciones psicológicas, cualitativamente peculiares, que surgen hacia el final de dicho periodo. (apud Bozhovich 1985, p. 123)
\end{abstract}

El concepto de situación social del desarrollo nos evidencia la preocupación de Vigotski por integrar la riqueza de los procesos internos, constituidos en la historia anterior del sujeto, con las influencias que caracterizan cada uno de los momentos sociales del desarrollo. En esa combinación, Vigotski se representa el vínculo constante de lo interno y lo externo en cada uno de los nuevos momentos cualitativos de este proceso, rompiendo así con la visión dicotómica, dominante hasta hoy en la psicología, sobre la relación entre lo externo y lo interno. Cada nueva situación social que el sujeto enfrenta se convierte en una via de desarrollo para nuevas formaciones psicológicas, término que no aparece elaborado de forma consistente en la obra de Vigotski, pero que luego aparece como uno de los temas centrales de los trabajos de L.I. Bozhovich, una de sus colaboradoras y seguidoras.

Antes de plantearse el significado como unidad de la conciencia, Vigotski se plantea la vivencia como unidad del desarrollo, como unidad de la situación social del desarrollo, entendiendo por vivencia la relación afectiva del niño con su medio. Para él, en la vivencia están representados tanto el medio, como lo que el niño aporta a través del nivel ya alcanzado por él, por tanto, la vivencia representa la unidad indisoluble de elementos externos e internos, que se expresan indisolublemente integrados en aspectos cognitivos y afectivos. La integración de lo cognitivo y lo afectivo es una idea presente de una u otra forma en muchos trabajos de Vigotski, lo que expresa su preocupación con el desarrollo de una metateoría psicológica con capacidad integradora sobre los temas y categorias que se habían estudiado de forma fragmentada dentro de la psicología.

La representación de Vigotski sobre la unidad de lo cognitivo y lo afectivo, le lleva a comprenderla como un proceso cualitativo diferente, lo cual se expresa claramente cuando afirma: 
Comprender la peculiaridad del niño retrasado mental significa, en primer lugar, que es necesario no sólo trasladar el centro de atracción del defecto intelectual hacia los defectos en la esfera afectiva; esto significa, en primer lugar, que es necesario elevarse, en general, sobre el análisis metafísico aislado del intelecto y del afecto, como esencias independientes, reconocer su vínculo interno y su unidad, liberarse del punto de vista sobre la relación del intelecto y el afecto como dependencia unilateral mecánica del pensamiento, del sentimiento.

En realidad, reconocer que el pensamiento depende del afecto significa hacer algo: virar al revés la teoría de T.F. Gerbardt, quien dedujo, de las leyes del pensamiento, la naturaleza del sentimiento. Con el fin de avanzar más, es necesario hacer lo que siempre ha sido una tradición indispensable del paso del estudio metafísico al estudio histórico de los fenómenos: es necesario analizar las relaciones entre el intelecto y el afecto que forman el punto central de todo el problema que nos interesa no como una cosa, sino como un proceso. (1989, p. 227)

En el párrafo analizado, se expresa claramente que Vigotski otorga a la emoción un lugar equivalente al de los procesos cognitivos en la constitución de las unidades constitutivas de la psique que, aunque no llega a explicitarlas en la complejidad en que se las plantea, evidencian un nivel diferente en la comprensión de lo psíquico, enfatizando la naturaleza procesual de sus formas más complejas de organización.

Al otorgarle a la emoción un status similar al de la cognición en la constitución de los diferentes procesos y formas de organización de la psique, Vigotski se está planteando la independencia de las emociones, en su origen, de los procesos cognitivos, e integrando las emociones dentro de una visión compleja de la psique que representa un importante antecedente para la construcción teórica del tema de la subjetividad. Esta visión compleja aparece de forma totalmente explícita en su crítica a la comprensión de K. Lewin sobre los procesos afectivos, en relación a lo cual expresa:

Los procesos afectivos que surgen en las necesidades verdaderas y no verdaderas ${ }^{2}$ y la tendencia dinámica motriz relacionada con ellos son analizadas por Lewin como algo inicial y que no dependen de la vida psíquica en general. Toda la vida psíquica depende de su base dinámica. Pero Lewin no ve el segundo aspecto de la dependencia, 
de que la propia base dinámica varía en el curso del desarrollo de la actividad física y, a su vez, pone de manifiesto la dependencia de las variaciones que sufre la conciencia en general. (1989, p. 217)

Realmente la emoción deja de ser un microconcepto sustancializado en definiciones aisladas y estáticas, centradas en tipos concretos de emoción, impulsos, necesidades, u otras formas concretas para conceptualizar sistemas de emociones consideradas como universales en el sujeto. Vigotski ve la emoción comprometida con el proceso cambiante de necesidades que va acompañando el desarrollo psíquico, elaboración de gran complejidad, que tendría que ser retomada y desarrollada con base en su obra.

En la cita anterior, puede verse con claridad el lugar constituyente otorgado por Vigotski a la base dinámica en la organización de los procesos psíquicos, así como su representación compleja de la psique en el individuo, la que apunta con toda claridad a comprender esta como proceso en desarrollo, que no se diluye ni en la acción del sujeto, ni en sus relaciones con los otros, ni en los lenguajes o voces que se producen en los escenarios sociales de su acción.

\section{La categoría de necesidad en la obra de Vigotski y otros autores marxistas: su valor heurístico en el estudio de las emociones}

Pensamos que una de las razones por las cuales el estudio de las emociones no ha tenido un progreso sensible dentro de la psicología es precisamente la ausencia de un marco teórico que permita integrarlas organicamente al proceso de producción de conocimiento psicológico, lo cual es uno de los objetivos que acompañan el desarrollo del tema de la subjetividad en nuestros trabajos actuales. Vigotski en varios momentos de su obra se refiere al término de necesidad, que consideramos una de las categorias importantes para el desarrollo del tema de las emociones y de la afectividad en general.

En uno de sus primeros trabajos Vigotski considera que "las emociones son el resultado de la apreciación que hace el propio organismo de su relación con el medio" (1926, p. 110). Para él las emociones surgían en minutos críticos del organismo, en momentos en que el equilibrio del organismo y el medio se rompía de una u otra forma (Bozhovich 1985). Sin 
embargo, ese concepto de periodo crítico, que Vigotski utiliza también en un determinado momento con el objetivo de desarrollar una periodización del desarrollo, ${ }^{3}$ no agota su representación sobre las necesidades que, como vimos, las ve como procesos que acompañan el desarrollo.

Vigotski plantea el tránsito de las necesidades biológicas a las necesidades sociales, propiamente humano, a partir de los primeros momentos del proceso de desarrollo, por tanto, él reconoce el desarrollo de las necesidades como un proceso especificamente humano, con lo cual supera la arraigada tendencia a definir la necesidad como concepto biológico. En relación al carácter social de las necesidades humanas, él escribe: "De aquí se comprende por qué cualquier necesidad del lactante, no importa cual sea, se convierte para él, en el curso del proceso de desarrollo, en una necesidad de otra persona, de contacto con el ser humano, de comunicación con él" (1985, p. 153). ${ }^{4}$

Vigotski utiliza con frecuencia la categoría de necesidad, a la cual da un status social en el humano, similar al que otorga a los otros proceso psíquicos, por tanto, la categoría forma parte de su arsenal conceptual en la organización de sus ideas sobre la psique. A pesar de que, por lo corta de su vida, y la abundancia de sus ideas, nos encontramos que la categoría no alcanza un nivel de madurez dentro de sus trabajos, lo cual puede ser definido como una característica general de su obra, que es en realidad una obra inconclusa. La muerte de Vigotski se produce en pleno desarrollo de su pensamiento.

Bozhovich escribe:

De esta forma, el análisis de la situación social del desarrollo del lactante llevó a Vigotski a la conclusión de que, desde los primeros días de vida, en el niño se dan las condiciones objetivas del desarrollo que crean las premisas necesarias para el surgimiento de la necesidad social, tanto por su contenido, como por su origen, de comunicación, y que esta necesidad constituye la base y la fuerza motriz de todo el desarrollo psíquico ulterior del niño. (1985, p. 153)

De acuerdo con esta autora, la necesidad de comunicación está en la base del desarrollo de las otras formas de necesidades superiores que aparecen en la ontogenia.

Con base en lo expuesto, podemos afirmar que el desarrollo de las necesidades superiores está comprometido, para Vigotski, con la cualidad 
del proceso de comunicación, con las emociones específicas y diferenciadas que se desarrollan en este proceso (lo subrayado son ideas del autor de este artículo). Es interesante que esta afirmación de Vigotski encontró un cuestionamiento en L.I. Bozhovich, quien se preguntó: (...) si la necesidad de comunicación surge en el proceso de la vida del niño, y no le ha sido dada desde el principio, cabe preguntarse cómo y sobre la base de cuáles necesidades primarias ella surge." (1985, p. 153)

La pregunta anterior que Bozhovich se plantea es esencial, pues a pesar de lo avanzado de su pensamiento en relación al tema de la afectividad, ella coloca la cuestión como si las necesidades superiores tuvieran que tener su origen en la "materia prima" de las necesidades primarias, lo cual actúa como presupuesto que le impide comprender lo que, para mí, es la base para el entendimiento en una nueva dimensión la necesidad: las necesidades no son entidades organizadas sobre una base orgánica definida, sino sistemas de emociones que se integran, generando estado dinámicos que, siempre, están relacionados con el funcionamiento integral del organismo, por lo cual siempre tienen una expresión fisiológica.

Sin embargo, la comprensión de la necesidad como momento permanente de la cualidad del organismo, especificada por su condición subjetiva y no biológica, fue de difícil acceso para los psicólogos de la época, así, A.N. Leontiev también comprendió la necesidad psicológica en analogía con la biológica: como estado de carencia. En este sentido escribió: "La necesidad es, en sí misma, como condición interna de la actividad del sujeto, sólo el estado negativo, el estado de ansiedad, de insuficiencia, su característica positiva sólo la recibe como resultado del encuentro con el objeto" (1974, p. 5). En esta definición se asume el concepto de necesidad primaria como ontología de la necesidad, analizándose su carácter cultural solamente por el tipo de objeto en que la necesidad se satisface, con lo cual se mantiene la comprensión de la necesidad dentro de un esquema homeostático de reducción de tensión, similar al defendido por Freud.

El tema de las necesidades fue, sin embargo, muy debatido dentro de la literatura marxista de los entonces países socialistas europeos. En este sentido, fueron muy interesantes las contribuciones del filósofo bulgaro L. Nikolov, quien señalo: "Quien asuma que la necesidad sólo tiene lugar allí donde el organismo se encuentra en un estado de ruptura de equilibrio, producto de la ausencia de determinada condición, también debe asumir que con la salida del organismo de ese estado desaparece la necesidad." (1982, p. 70) 
Este cuestionamiento tiene gran importancia, en el sentido que nos remite a reflexionar sobre la necesidad, no como algo aislado, sino como momento cualitativo de un sistema en desarrollo que, de hecho, coincide con la perspectiva más general desarrollada por Vigotski en relación a este término. L. Nikolov expresa de forma explícita que:

La "necesidad" es la nominación generalizada, en esencia, de tres cualidades del organismo vivo que se complementan consecuentemente entre sí:

En primer lugar, la cualidad más fundamental general del organismo vivo: la cualidad de funcionar por determinado programa, en calidad de los parámetros dados en el programa de ocurrencia del proceso vital; parámetros que abarcan las relaciones entre los procesos vitales en su conjunto y los distintos momentos que los integran.

En segundo lugar, la cualidad del organismo de reaccionar al estado existente de relaciones indicado más arriba (intranquilidad o ansiedad ante la amenaza de ausencia o la ausencia real de los correspondientes momentos condicionales, como la satisfacción, tranquilidad, goce y alivio ante la existencia de las posibilidades de su garantía, ante su inclusión real en el funcionamiento normal).

En tercer lugar, la cualidad del organismo de motivar y movilizar las formas más diversas de actividad, con ayuda de las cuales garantiza el funcionamiento normal del correspondiente proceso vital. (1982, p. 74)

En las reflexiones de Nikolov se presenta la necesidad como una cualidad del organismo que mantiene viva su capacidad como sistema para actuar y seguir un programa que garantiza su proceso vital. En este sentido el autor nos presenta una definición amplia, válida para todos los organismos vivos, en la que enfatiza la necesidad como momento del proceso vital.

Esta definición es legitima para las necesidades inherentes a los sistemas vivos, y en ella se enfatiza el compromiso de la necesidad con el funcionamiento integral del sstema. Sin embargo, cuando los procesos psíquicos adquieren carácter cultural, y se desarrollan los procesos psíquicos superiores que Vigotski nos presenta, sobre cuya concepción aparece una nueva representación sobre la especificidad ontológica de la psique en el hombre, comprometida con su naturaleza cultural, el hombre pasa a tener nuevas necesidades, que se especifican a nivel subjetivo, 
pero que acompañan y caracterizan toda su constitución como sistema, incluyendo los procesos biológicos.

Podemos afirmar que el sujeto actúa en estados de necesidad y que, en su actuación, mantiene una capacidad generativa permanente de nuevas necesidades, que participarán en el sentido subjetivo de sus diferentes actividades, estados y experiencias. En el nivel subjetivo, esas nuevas necesidades se organizan a través de emociones ya existentes, las que tienen procedencias diferentes, y que, en su diversidad, y por su fragmentación, se integran en la definición de nuevos estados cualitativos del organismo, que aparecen como nuevas necesidades.

La aparición de nuevas necesidades psicológicas es fuente de emociones cualiativamente diferentes, que en sus nuevas vías de integración con otras emociones en momentos concretos de la actividad, y del estado general del sujeto, conducirán a la aparición de nuevas necesidades. El proceso de aparición de nuevas necesidades y emociones es inseparable del comportamiento del organismo como sistema, que, en el caso de los seres humanos, es un sistema complejizado por su condición subjetiva, que incluye su capacidad de acción diferenciada como sujeto de sus propias actividades.

Esta complejidad sistémica del organismo humano, y su significación para el estudio de los procesos afectivos, Vigotski se la planteó ayudado por su posición dialéctica frente al problema, premisa que le favoreció su representación procesual y compleja sobre las funciones psíquicas, la cual, aunque él no consiguió explicitarlo, constituyó una importante premisa de la construcción de los procesos psíquicos como subjetividad. En análisis anteriormente citado, donde estudia la concepción dinámica de Lewin, Vigotski escribe:

El desconoce (se refiere a Lewin) la regla dialéctica de que, en el curso del desarrollo, la causa y las consecuencias cambian de lugares, que una vez que han surgido las formaciones psíquicas superiores, sobre la base de ciertas premisas dinámicas, ejercen una influencia retroactiva en los procesos que las originaron; que en el desarrollo lo más bajo cambia por lo más alto, que en el desarrollo cambian no sólo las funciones fisiológicas por sí mismas, sino que en primer lugar varían los nexos interfuncionales y las relaciones entre los diferentes procesos, en particular, entre el intelecto y el afecto. Lewin analiza el afecto al margen del desarrollo y al margen de la relación con la vía psíquica restante. (1989, p. 217) 
Como hemos observado en el curso del presente epígrafe, el afecto, construido a través de las categorias de necesidades y emociones, así como a partir de los sentimientos, es un aspecto constituyente esencial del sistema psíquico, dentro del cual eses procesos, tanto los afectivos como los cognitivos, no funcionan de modo aislado, sino como momentos de un sistema complejo, que Vigotski coloca en diferentes momentos de su obra, en el desarrollo, la personalidad y la conciencia. En general, Vigotski consigue integrar los temas de conciencia y desarrollo; sin embargo, a pesar de haber tenido muy pocas referencias en relación a la cuestión de la personalidad, en esas pocas referencias nos presenta un concepto diferente de personalidad que, como veremos más adelante, está libre de invariantes universales.

Como afirmamos anteriormente, Vigotski es un autor que nos legó una obra inconclusa, sobre lo cual, con motivo de los 100 años de su nacimiento, presenté un artículo donde enfatizaba la diferencia entre lo que considero la teoría más orgánica de Vigotski, y su representación general sobre la psicología, la cual él no consigue sistematizar en ninguna de sus obras, y aparece de forma dispersa a lo largo de estas. ${ }^{5}$ Precisamente por ese carácter fragmentado y apurado en el tratamiento de algunos temas, muchas de las cuestiones tratadas en el presente artículo aparecen de forma contradictoria en diferentes momentos de su propia obra, lo cual hace difícil seguir los términos y ubicarlos con claridad.

Un ejemplo de las contradicciones que señalamos en el párrafo anterior es la relacionada con el tratamiento que dio al término vivencia en el estrecho periodo de tiempo en el que concentró su atención sobre el mismo. Después de definir la vivencia como unidad de lo externo y lo interno, y de lo afectivo y lo cognitivo, terminó definiendo la vivencia por la capacidad de generalización del niño, lo cual representó un reduccionismo cognitivista, que entra en contradicción con la mayoría de las citas de sus trabajos que hemos utilizado en el presente artículo.

L.I. Bozhovich escribe: "La tesis de L.S. Vigotski, acerca de que, en fin de cuentas, la vivencia se determina por el nivel de desarrollo de las generalizaciones, es decir, por la interpretación, es de hecho también errónea" (1985, p. 125). Y más adelante expresa:

(...) tras la vivencia se encuentra el mundo de las necesidades del niño - de sus aspiraciones, deseos, propósitos - en su complejo entrelazamiento y en su correlación con las posibilidades de 
satisfacción. Y todo este complejo sistema de vínculos, todo este mundo de necesidades y aspiraciones del niño debe ser descifrado para comprender el carácter de la influencia de las condiciones externas sobre el desarrollo psíquico del niño (1985, p. 128).

El reconocimiento de la relación entre necesidades y emociones, así como el lugar que Vigotski otorga a las formaciones psicológicas superiores, nos remiten a un sistema de naturaleza social, pero constituido en el individuo, que es el escenario complejo en que estas relaciones tienen lugar en el sistema de la vida psíquica del sujeto: la personalidad.

\section{La significación de la personalidad en la comprensión de la organización de los procesos afectivos como procesos psíquicos superiores}

El tema de la personalidad se asocia hasta hoy con los sistemas teóricos desarrollados en la clínica, lo cual ha sido una de las razones de su exclusión de áreas como la psicología social y, aunque en menor grado, de la propia psicología educacional. Este rechazo es debido a la representación sustancializada de la personalidad, comprendida a través de categorías sustentadas en una visión del hombre que acaba por imponer sus términos a cualquier función o proceso específico que sea estudiado. En nuestra opinión, el valor heurístico de la personalidad viene dado por su capacidad integradora del funcionamiento de la subjetividad individual, y no por sus contenidos psicológicos concretos.

Tanto Vigotski como Rubinstein presentaron una comprensión de la personalidad como sistema básico para la construcción del pensamiento psicológico, en la cual ésta no aparecía sustancializada en sus atributos concretos, sino que representaba un sistema de organización y funcionamiento de la psique en el individuo, con lo cual se reconocía el status de la constitución psíquica en el individuo y, a su vez, se separaba el concepto de personalidad de una representaión universal sobre lo individual, la cual fue carcaterística de todas las aproximaciones dinámicas a su estudio.

En nuestra opinión la personalidad representa el nivel constitutivo de la subjetividad individual, donde las necesidades manifiestan sus formas subjetivas de expresión diferenciada en los motivos. Durante mucho tiempo las categorías de motivo y necesidad fueron empleadas práctica- 
mente como sinónimos, como momentos de una taxonomía organizada de microconceptos de naturaleza y valor heurísticos similares. Eran, en muchas de las taxonomías presentadas por diferentes teorías psicológicas, sobre todo aquellas de carácter empírico, simplemente categorías definidas a través de formas comportamentales, que no tenían ningún vínculo con construcciones teóricas más amplias y profundas.

Las necesidades humanas se integran entre sí a través de innumerables sentidos subjetivos y configuran los motivos. Todo motivo es una combinación particular de sentidos subjetivos que, organizados en las más diversas áreas de actividad humana, representa una integración cualitativa nueva, donde la necesidad constituyente fundamental se integra cualitativamente en una nueva unidad, que define la producción de sentidos subjetivos en los diferentes espacios de la vida del sujeto. El nivel diferenciado de producción de sentidos subjetivos a partir de una necesidad se da por su conversión en motivo.

La necesidad sexual, por ejemplo, deviene motivo en el proceso de integración de otros sentidos subjetivos provenientes de otras esferas de la vida psíquica que pasan a ser constituyentes del motivo sexual, como la moral, la constitución de género, la cualidad de las diferentes relaciones emocionales de la persona a lo largo de la vida, el sentido y significación del cuerpo etc. Todas estas dimensiones de sentido se organizan en el motivo sexual y caracterizan el tipo de emociones asociados a él. Estos complejos sistemas de motivos articulados a las diferentes formas de acción del sujeto los hemos denominado configuraciones de la personalidad, y constituyen el tipo de unidad fundamental, que hoy empleamos en el estudio de la personalidad.

La personalidad representa la conceptualización de un sistema responsable por la legitimación de lo singular en la constitución de la subjetividad. Vigotski escribe en relación a la personalidad:

El problema de la insuficiencia motriz es un bello ejemplo de aquella unidad en la heterogeneidad observada en el desarrollo del niño con defecto. La personalidad se desarrolla como un todo único, como un todo único reacciona ante la deficiencia, ante la alteración del equilibrio originada por ella y forma un nuevo sistema de adaptación y un nuevo equilibrio en lugar del alterado. Pero precisamente debido a que la personalidad representa una unidad y actúa como un todo único, ésta, en el desarrollo, hace avanzar de un modo desproporcional unas $u$ otras funciones diversas y rela- 
tivamente independientes una de las otras. Estos postulados, es decir, la variedad de las funciones relativamente en el desarrollo y la unidad del todo en el proceso de desarrollo de la personalidad, además de no contradecirse unos a los otros, como lo demostró Stern, se condicionan recíprocamente. (1989, p. 14)

Podemos apreciar como Vigotski utiliza el concepto de personalidad para designar la integración dialéctica y en desarrollo de funciones diferentes, que alcanzan niveles de integración cualitativa y única como sistema, en un nivel del funcionamiento psicológico, que define como personalidad, con lo cual pienso que integra la categoría a un campo básico de producción teórica, que simplemente no existía en la psicología, y que hasta hoy todavía no ha sido institucionalizado, en tanto el término psicología básica continúa asociado a la investigación experimental en muchas de las universidades del mundo.

Vigotski tenía una representación clara del carácter social e histórico de la personalidad humana, y se representaba ésta como un momento importante de sentido subjetivo, para oponerse a concepciones fatalistas en relación al impacto de los defectos psíquicos en la definición de la personalidad humana, en relación con lo cual tomó partido al escribir: "La psicología de la personalidad rompe definitivamente con el estatismo biológico en el enfoque del carácter (...) ya que en contraposición a la teoría de Freud, en lugar de la fatalidad biológica pone las fuerzas formadoras y motrices de la historia y de la vía social (...). En resumidas cuentas, el defecto por sí solo no decide el destino de la personalidad"... (1989, p. 30)

La personalidad no representa una entidad intrapsíquica sutancializada para Vigotski, sino un sistema complejo integrador de la vida psíquica individual, que participa en el sentido que las experiencias tienen para el sujeto. De esta manera, Vigotski utiliza el término en el campo de la educación especial, donde después de él prácticamente el término no ha sido usado nuevamente en el desarrollo teórico concreto que esta esfera presenta.

El tema de las emociones no puede encontrar un tratamiento consecuente si es tratado de forma aislada, fuera de las dimensiones de sentido de la constitución subjetiva individual que participan en la definición del sentido de las diferentes emociones producidas por el hombre. La personalidad es una construcción teórica, que reconoce ontológicamente la subjetividad individual, solo que no se representa ésta desde una visión 
esencialista y mecanicista, como entidad separada de lo social, sino en la procesualidad de un sujeto que existe socialmente, y cuya personalidad tiene una naturaleza social e histórica.

El rescate de estas categorias representa una necesidad de una psicología comprometia con el legado de Vigotski, el cual con frecuencia ha sido deformado por interpretaciones reduccionistas, que pretenden colocar de forma unilateral el tema de lo psicológico en el nível social o en el de las significaciones, no atendiendo a su complejidad dialéctica en la superación de las dicotomías que Vigotski intento derrumbar el tiempo todo.

\section{Notas}

1. Para mayor información sobre estas críticas ver Psicología de la personalidad. Habana, Pueblo y Educación, 1985; Psicologia principios y categorias. Habana, Editorial de Ciencias Sociales, 1989; y Personalidad, comunicación y desarrollo. Habana, 1995.

2. Aquí se está refiriendo al concepto de "cuasi necesidad", desarrollado posteriormente por su discípula B. Zeigarnik dentro de la psicología soviética.

3. Ver "El problema de la periodización por edades del desarrollo infantil". En Tomo 7, Obras Completas. Moscú, Editorial Pedagógica, 1984.

4. Esta cita fue tomada del libro de L.I. Bozhovich, La personaliad y su formación en la edad infantil, y la referencia es de un trabajo no publicado de Vigotski titulado "La edad de la lactancia".

5. El artículo de referencia es: "L.S. Vigotski: presencia y continuidad de su pensamiento en el centenario de su nacimiento". Psicologia \& Sociedade, ㄲo 2 , vol. 8. São Paulo, 1996.

\section{The place of emotions in the psychic social constitution: Vygotsky's contribution}

ABSTRACT: The article analyses the place of emotions in the psychic social constitution, highlighting, overall, the Vigotsky's contribution. The text focus on two of the main ideas which caused an impact in the psychology reconstructions by Vygotsky: the psychic functions in the activity and the signals mediation in the constitution of the functions. The concept of "social situation development" reveals Vygotsky's preocupation to integrate the richness of the internal processes, constituted in the former subject history, with the influences that give 
the characteristic to each of the social moments of the development. We also focus on the importance that the "experience" and the "necessity" category has in Vygotsky's thought. At last, it's analized the "personality" issue as a theoretical construction that recognizes ontologically the individual subjectivity, understood as a process of a subject that exists socially.

\section{BIBLIOGRAFA}

BOZHOVICH, L.I. La personalidad y su formación en la edad infantil. Habana: Pueblo y Educación, 1985 (versión original en ruso, 1968).

NIKOLOLOV, L. Estructura de la actividad humana. Moscú: Progreso, 1982.

GONZÁLEZ R., Fernando. Psicología de la personalidad. Habana: Pueblo y Educación, 1985.

. Psicología: Principios y categorias, Habana: Pueblo y Educación, 1989.

VYGOTSKY, Lev S. Psicología pedagógica, Moscú: Prosveschenie, 1926. . Desarrollo de las funciones psíquicas superiores. Moscú: Editorial de la Academia de Ciencias Pedagógicas, 1960.

. El Problema del retraso mental, Obras Completas, tomo 5. Habana: Pueblo y Educación, 1989.

. Problemas fundamentales de la defectología. Obras Completas, tomo 5. Habana: Pueblo y Educación, 1989. 